\title{
Preface
}

\section{Rhinoplasty: A Complex, Four-Dimensional Procedure}

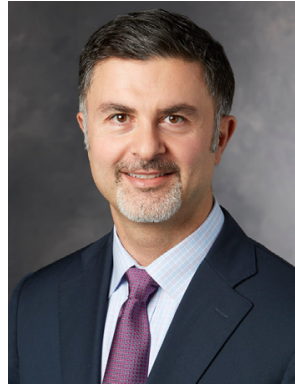

Sam P. Most, MD, FACS

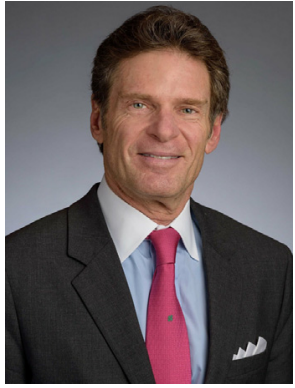

Alan Matarasso, MD, FACS

Editors

Rhinoplasty is widely accepted as one of the most, if not the most, challenging procedures in plastic surgery, comprising numerous interconnected maneuvers that have immediate and long-term consequences on aesthetics and function. Rhinoplasty requires mastering reshaping a threedimensional bony and cartilaginous structure that projects from the face, taking into account the effect of the skin-soft tissue envelope on results. Finally, the fourth dimension of rhinoplasty surgery, the changes that occur over time, requires the long view in determining success or failure in our results. The results are scrutinized, "front and center," for patients' entire lives. As such, it is fair to say that rhinoplasty impacts appearance perhaps more than any procedure we do.

While John Orlando Roe, an otolaryngologist in Michigan, was the first to publish an article describing this operation in 1887, it was Jacques Joseph, an orthopedic surgeon by training and considered a father of plastic surgery, who pioneered and popularized many of the techniques and instruments used in rhinoplasty in the early twentieth century. Indeed, most common dorsal hump reduction methods are derived from his concepts. In the mid-twentieth century, Maurice Cottle championed concepts of the central importance of nasal physiology and popularized another method for dorsal hump reduction, involving impaction of the dorsum into the maxilla. Many of the leaders in the field of rhinoplasty, plastic surgeons, otolaryngologists, and facial plastic surgeons, continued to develop and teach methods of rhinoplasty from these early pioneers. Over the past century and more, countless articles and textbooks have been published on the art and science of this operation.

So, why the need for yet another compilation on this well-described procedure? Simply put, the bar for measuring success of our results in the procedure is constantly rising. As masters of this operation have shared their experience, our 
understanding of associated complications and corrective measures has increased. One obvious example would be Jack Sheen's understanding of the importance of midvault reconstruction after dorsal hump resection. Another example: the idea that one could use the excess upper lateral cartilages as spreader grafts was first described by Fomon in the 1950s but was not popularized for another 50 years.

In the past decade, new ideas have been added to our armamentarium. The use of powered instrumentation has gained acceptance. We are moving from minimizing removal of alar cartilages to complete alar preservation during tip plasty. And, of course, the concept of dorsal preservation, over 100 years old, is now making a comeback.

Countless other examples exist and are constantly being added to our literature. We are constantly evolving our practice to improve outcomes for our patients. As such, we have attempted in this issue to update our readers on some of the advances in rhinoplasty concepts and practice. This issue of Clinics in Plastic Surgery has something for rhinoplasty surgeons of all levels of interest and experience. We hope this brings all readers another step closer to improving our rhinoplasty outcomes and patient satisfaction. We would like to thank our authors, all esteemed members of the community of rhinoplasty surgeons, for their contributions to this issue.

Sam P. Most, MD, FACS

Division of Facial Plastic and Reconstructive

Surgery

Stanford University School of Medicine

801 Welch Road

Stanford, CA 94304, USA

Alan Matarasso, MD, FACS

Hofstra-Northwell Health System

New York, NY 10028, USA

E-mail addresses:

Smost@stanford.edu (S.P. Most)

amatarasso@drmatarasso.com (A. Matarasso) 